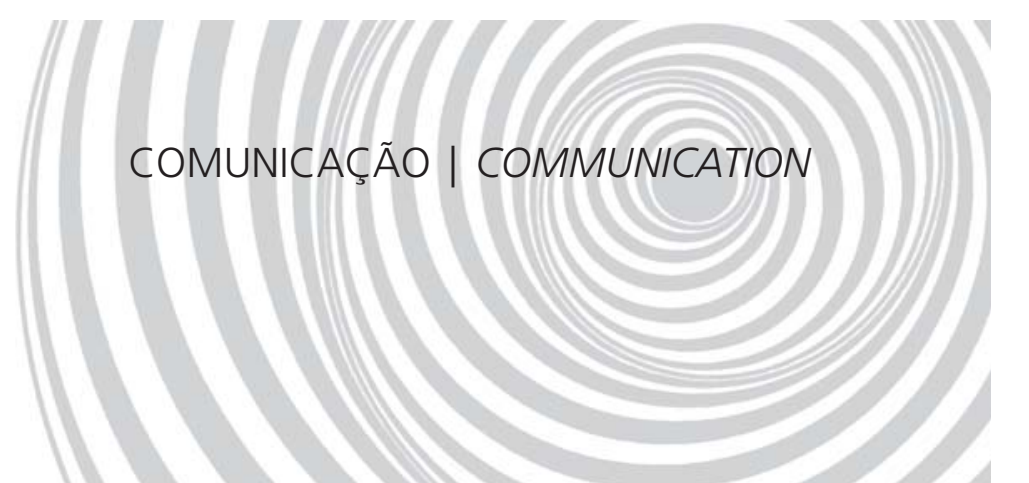

\title{
Índice glicêmico: uma abordagem crítica acerca de sua utilização na prevenção e no tratamento de fatores de risco cardiovasculares
}

\author{
Glycemic index: a critical analysis of its use as a tool \\ to prevent and treat cardiovascular risk factors
}

Gisele Queiroz CARVALHO'

Rita de Cássia Gonçalves ALFENAS ${ }^{1}$

RESU M O

As doenças cardiovasculares são consideradas um grande problema de saúde pública, uma vez que são a principal causa de morte no Brasil e no mundo. Essas doenças possuem etiologia multifatorial. Além da susceptibilidade genética, outros fatores, tais como idade e presença de hipertensão arterial, dislipidemias, obesidade, diabetes mellitus, inatividade física e alguns hábitos alimentares inadequados, são considerados importantes fatores de risco para o desenvolvimento de doenças cardiovasculares. O elevado consumo de carboidratos tem sido associado ao aumento da obesidade, às dislipidemias, à intolerância à glicose/diabetes mellitus e à resistência insulínica, estando, dessa forma, entre os fatores de risco das doenças cardiovasculares. Entretanto, existem controvérsias acerca da influência da qualidade do carboidrato ingerido no desenvolvimento de tais doenças, independentemente da quantidade ingerida. O índice glicêmico é um indicador da qualidade do carboidrato ingerido. Sua utilidade em condições de vida livre tem sido questionada, devido à interferência de vários fatores, os quais são difíceis de serem controlados sob tais condições. Este trabalho objetivou analisar criticamente os estudos que avaliaram o efeito do índice glicêmico dos alimentos na manifestação de doenças cardiovasculares e seus fatores de risco.

Termos de indexação: Índice glicêmico. Doenças cardiovasculares. Aterosclerose. Dislipidemias. Carboidratos.

\section{A B S T R A C T}

Cardiovascular diseases are considered a great public health problem since they are the main cause of death in Brazil and in the world. These diseases have a multifactorial etiology. Besides genetic susceptibility, other factors such as age, hypertension, dyslipidemia, obesity, diabetes mellitus, physical inactivity and some inadequate dietary habits are considered important risk factors for the development of cardiovascular diseases. However, there are several controversies concerning how the quality of the ingested carbohydrate influences

\footnotetext{
${ }^{1}$ Universidade Federal de Viçosa, Departamento de Nutrição e Saúde. Av. PH Rolfs, s/n., Campus Universitário, 36570-000,

Viçosa, MG, Brasil. Correspondência para/Correspondence to: R.C.G. ALFENAS. E-mail: <ralfenas@ufv.br>.
} 
578 | G.Q. CARVALHO \& R.C.G. ALFENAS

the development of such diseases, regardless of amount. The glycemic index is an indicator of carbohydrate quality. Its use in free living conditions has been questioned, due to the interference of several factors which are difficult to control under such conditions. The aim of this paper was to critically analyze studies that evaluated the effect of the glycemic index of foods in the manifestation of cardiovascular diseases and its risk factors.

Indexing terms: Glycemic index. Cardiovascular diseases. Atherosclerosis. Dyslipidemias. Carbohydrates.

\section{N T R O D U ÇÃ O}

O aumento da prevalência de doenças cardiovasculares tem preocupado os especialistas no Brasil e em vários países do mundo, uma vez que as doenças do aparelho circulatório são indicadas como a primeira causa de morte em relação às demais, gerando enormes gastos em saúde pública ${ }^{1-3}$. Segundo Santos Filho \& Martinez $z^{4}$, as doenças cardiovasculares respondem por 30\% das mortes em todas as faixas etárias. Estima-se que a prevalência de angina nos paises europeus seja de 20 a 40/1000 habitantes, considerando ambos os sexos 5 . Na Escócia essa prevalência é de 28/1000 em homens e de 25/1000 em mulheres, aumentando com a idade e a condição socioeconômica ${ }^{6}$.

A elevada incidência dessas doenças parece ser um reflexo da transição nutricional, a qual é caracterizada pela diminuição dos casos de desnutrição e o aumento dos casos de sobrepeso/ obesidade, evidentes em todo o mundo, e o Brasil tem acompanhado essas tendências. Esta mudança no perfil nutricional da população é resultante da diminuição da prática de atividade física de lazer e de mudanças no padrão alimentar da população, caracterizadas por aumento do consumo de gorduras (principalmente saturadas, de origem animal), açúcar e alimentos refinados, e pela redução do consumo de carboidratos complexos e de fibras ${ }^{7-9}$.

As doenças ateroscleróticas possuem etiologia multifatorial. Além da susceptibilidade genética e da idade, a hipertensão arterial, as dislipidemias, a obesidade, o diabetes mellitus e alguns hábitos de vida são considerados importantes fatores de risco para o desenvolvimento de doenças cardiovasculares ${ }^{1,3,10}$. Dessa forma, a diminuição da exposição ou a remoção dos fatores de risco são pontos importantes a serem considerados para a redução da mortalidade e/ou redução da prevalência e/ou para o surgimento mais tardio de doenças ateroscleróticas, isquêmicas e cerebrovasculares ${ }^{10}$.

Entre os principais hábitos de vida envolvidos no desenvolvimento dessas doenças podese citar o tabagismo, o sedentarismo e o consumo de dieta com alto teor energético, rica em gorduras (principalmente a saturada), colesterol e sal ${ }^{1,11}$. A prática de atividade física e as mudanças no hábito alimentar têm sido os principais fatores envolvidos na prevenção ou na melhora dos fatores de risco das doenças cardiovasculares ${ }^{3}$.

Com relação à nutrição, tem sido observado que além da ingestão excessiva de energia, os componentes da dieta possuem grande influência no desenvolvimento ou na prevenção dessas doenças. Neste sentido, os aumentos da ingestão de ácidos graxos saturados, ácidos graxos trans e colesterol dietético são associados ao aumento de colesterol total, em especial ao aumento da lipoproteína de baixa densidade (LDL) ${ }^{3,5}$. Os ácidos graxos trans, ainda, promovem diminuição da lipoproteína de alta densidade (HDL) ${ }^{12}$. Já os ácidos graxos monoinsaturados e poliinsaturados são considerados benéficos, uma vez que os primeiros são associados à diminuição de colesterol total e ao aumento da HDL, enquanto que os últimos são associados à diminuição dos triglicerídeos séricos, à melhora da função plaquetária e à diminuição da pressão arterial em hipertensos ${ }^{3,5}$.

As fibras, principalmente as solúveis, possuem efeito benéfico, reduzindo o colesterol total, a LDL e melhorando a tolerância à glicose. Também as substâncias antioxidantes da dieta, como vitamina $\mathrm{E}$, pigmentos carotenóides, vitamina C, flavonóides e outros compostos fenólicos, 
são associadas a tais benefícios, uma vez que estas aumentam a resistência da LDL à oxidação³.

A associação entre consumo de lipídeos e de fibras e desenvolvimento de doenças cardiovasculares é bastante conhecida e estudada. Entretanto, a influência do consumo de carboidratos ainda é bastante controversa. O elevado consumo de carboidratos tem sido associado ao aumento da obesidade, das dislipidemias, da intolerância à glicose, do diabetes mellitus e da resistência insulínica, estando dessa forma entre os fatores de risco para as doenças cardiovasculares ${ }^{13,14}$. Em relação ao perfil lipídico, o consumo das dietas com alto teor de carboidratos pode aumentar as concentrações de triglicerídeos plasmáticos e reduzir as concentrações de $\mathrm{HDL}^{15}$. Segundo alguns autores, as dietas restritas em carboidratos são mais benéficas que aquelas com baixo teor de lipídeos ${ }^{16}$. Entretanto, não há consenso se a qualidade do carboidrato ingerido pode ser um fator de risco para o desenvolvimento de tais doenças, independentemente da quantidade ingerida. Neste sentido, vários estudos tentam relacionar o índice glicêmico ao desenvolvimento de doenças cardiovasculares, ou à melhora dos parâmetros e fatores de risco para tais doenças. O índice glicêmico é um indicador da qualidade do carboidrato ingerido, e existem controvérsias quanto à sua utilização e à sua eficácia na dieta de indivíduos em condições de vida livre (ou seja, condição sem controle laboratorial) $)^{11,17-19}$.

Por meio desta comunicação foram analisados criticamente os estudos que avaliaram o impacto do índice glicêmico dos alimentos na manifestação de doenças cardiovasculares e seus fatores de risco.

\section{Índice glicêmico e doenças cardiovasculares}

O índice glicêmico foi desenvolvido por Jekins et al. em 1981, a partir da comparação dos efeitos fisiológicos de alimentos contendo carboidratos em relação à sua composição química. Este índice corresponde à classificação de um alimento em relação ao efeito que ele exerce na glicemia pós-prandial, em comparação àquela observada após o consumo de um alimento referência, ambos contendo a mesma quantidade de carboidrato disponível ( $50 \mathrm{~g}$ ou $25 \mathrm{~g}$ ), sendo testados em um mesmo indivíduo. O índice glicêmico de um alimento é expresso como a porcentagem da área abaixo da curva da resposta glicêmica pós-prandial do alimento teste, em relação a este mesmo tipo de resposta após a ingestão de um alimento padrão ${ }^{19,20}$.

Tanto o pão branco, quanto a glicose podem ser utilizados como alimento padrão para a determinação do índice glicêmico. Entretanto, devido às variações em sua composição entre os países ou mesmo entre as cidades dentro de um mesmo país, convencionou-se que a utilização da glicose anidra seria mais recomendada ${ }^{21}$.

O conceito de índice glicêmico foi proposto devido ao reconhecimento que diferentes alimentos contendo a mesma quantidade de carboidrato possuem diferentes efeitos fisiológicos ${ }^{19}$. Segundo alguns autores, alimentos com baixo índice glicêmico promovem menor elevação da glicemia pós-prandial, devido à sua lenta taxa de digestão e absorção. Por outro lado, os alimentos com alto índice glicêmico proporcionam um maior aumento da glicemia por serem digeridos e absorvidos mais rapidamente20,22.

Logo após sua criação, este índice passou a ser considerado como importante ferramenta no tratamento e no controle do diabetes mellitus. Sua utilização também foi sugerida para pacientes com doenças cardiovasculares, ou com risco de desenvolvimento destas ${ }^{23}$, uma vez que a redução da glicemia e da insulinemia pós-prandial é desejável no controle e na prevenção do desenvolvimento de tais doenças ${ }^{24}$. Diante disso, alguns estudos têm sugerido que a ingestão de alimentos de absorção lenta pelo intestino delgado favorece a redução do risco de desenvolvimento de doenças cardiovasculares ${ }^{20}$.

Existem duas hipóteses para explicar o mecanismo pelo qual o consumo de alimentos com alto índice glicêmico pode favorecer o 
aumento da manifestação de doenças cardiovasculares. A primeira hipótese baseia-se na elevação brusca da glicemia, resultando em períodos de hiperglicemia ${ }^{25}$. Tal hiperglicemia, mesmo em indivíduos não diabéticos, pode causar glicosilação das LDLs, tornando essas lipoproteínas lesivas ao endotélio. Esta lesão pode iniciar um processo inflamatório e progredir para o desenvolvimento de aterosclerose ${ }^{26}$. De acordo com Wolever \& Mehling ${ }^{24}$, o risco de desenvolvimento de doenças cardiovasculares aumenta continuamente com o aumento da glicemia além dos níveis normais.

A segunda hipótese se refere à elevação da insulinemia, como resultado da elevação glicêmica. De modo geral, a resposta insulinêmica se correlaciona à resposta glicêmica. Dessa forma, o índice glicêmico pode também afetar a insulinemia pós-prandial, embora essa relação não seja sempre tão direta ${ }^{20,25,27}$. A elevada resposta insulinêmica tem sido relatada como um dos mais importantes fatores que promovem o desenvolvimento de doenças cardiovasculares, já que a hiperinsulinemia predispõe ao desenvolvimento de dislipidemias, hipertensão arterial e disfunção endotelial. A hiperinsulinemia ainda se associa ao desenvolvimento do diabetes mellitus, devido à exaustão das células beta pancreáticas, e à obesidade, devido ao aumento do apetite e da ingestão alimentar. Todos esses fatores estão diretamente associados ao desenvolvimento de doenças cardiovasculares, sendo a hiperinsulinemia considerada um fator de risco independente para o desenvolvimento de doenças coronarianas ${ }^{25,28}$. Dessa forma, a redução da glicemia e da insulinemia pós-prandiais pode ser benéfica na prevenção e no tratamento da resistência insulínica, de modo a evitar o desenvolvimento de doenças cardiovasculares ${ }^{24}$.

No entanto, este é um assunto bastante controverso e as possíveis aplicações atribuídas ao índice glicêmico não são aceitas por toda a comunidade científica ${ }^{29}$. O índice glicêmico é aceito na Austrália, no Canadá e na Europa. Entretanto, sua utilização não é recomendada pela American Diabetes Association (ADA). A ADA destaca que a comprovação da aplicação e do impacto do índice glicêmico na prática clínica exige que mais estudos sejam $\operatorname{conduzidos}^{30}$. Segundo pesquisadores, o índice glicêmico ainda não é uma ferramenta aplicável à população, já que inúmeras variáveis favorecem sua alta variabilidade inter e intraindividual, interferindo na reprodutibilidade do mesmo ${ }^{17,18,27}$.

Vários fatores podem alterar a taxa de motilidade intestinal, a digestão e a absorção, resultando em diferenças no índice glicêmico dos alimentos. Os principais fatores que interferem nesse parâmetro incluem o estágio de maturação das frutas, a forma física (líquido, pastoso, sólido) apresentada pelo alimento, a composição do alimento (quantidade de carboidratos, proteínas, lipídeos, fibras, conteúdo de água), o tipo de amido (amilose, amilopectina), o tipo de processamento ao qual o alimento foi submetido, acidez, volume e temperatura do alimento ingerido, além do estado fisiológico apresentado pelo indivíduo 25,29,30.

Diante disso, a ADA destaca que tanto a quantidade, quanto a qualidade do carboidrato consumido podem ser importantes no controle da glicemia e das demais alterações metabólicas, sendo a quantidade o fator primário a ser controlado na dieta. A ADA ainda destaca quatro principais pontos que impossibilitam a utilização do índice glicêmico como único e mais importante instrumento no controle da glicemia ${ }^{30}$ :

- O índice glicêmico considera apenas o tipo de carboidrato, ignorando o efeito da quantidade total do carboidrato ingerido. Entretanto, ambos influenciam a resposta glicêmica pósprandial.

- O valor do índice glicêmico atribuído a determinado alimento apresenta grande variação, devido às variações intra e interindividuais na resposta glicêmica e em função das diferenças metodológicas utilizadas para sua determinação.

- As tabelas internacionais de índice glicêmico apresentam o valor desse parâmetro para alimentos ingeridos de forma isolada. Tais tabelas não prevêem as alterações desse valor, em função 
da ocorrência de interações entre os diversos tipos de nutrientes presentes nesses alimentos quando os mesmos são consumidos em refeições mistas.

- O índice glicêmico reflete de maneira mais precisa a resposta glicêmica de indivíduos normais, do que daqueles com diabetes.

Atualmente, considera-se que o índice glicêmico seja uma ferramenta relativamente reprodutível em condições laboratoriais, em que o indivíduo ingere uma quantidade fixa de carboidrato disponível. No entanto, em condições de vida livre, a quantidade de carboidrato ingerido em determinada refeição normalmente varia. Assim, na tentativa de minimizar os erros causados pela variação da quantidade de carboidratos consumidos em cada refeição, foi introduzido o conceito de carga glicêmica. Este conceito é derivado do índice glicêmico, levando em consideração a quantidade do carboidrato ingerido ${ }^{24,31}$. É expresso pela fórmula:

Índice glicêmico do alimento x Teor Carga glicêmica $=\frac{(\mathrm{g}) \text { de carboidrato ingerido }}{100}$

Para alguns autores, a utilização da carga glicêmica pode ser válida em condições de vida livre, já que considera a qualidade e a quantidade do carboidrato do alimento em um mesmo índice ${ }^{14}$. No entanto, por ser um derivado do índice glicêmico, a carga glicêmica está sujeita a todas as alterações e influências que ocorrem no índice glicêmico. Outros autores não consideram a carga glicêmica como ferramenta válida. Para esses autores, a qualidade do carboidrato (índice glicêmico) apresenta associação mais forte aos riscos de doenças (diabetes, doenças cardiovasculares e câncer) do que o conteúdo de carboidrato ou a carga glicêmica da dieta ${ }^{14,32}$.

\section{ESTUDOS EN VOLVENDO ÍNDICE GLICÊMICOE FATORES DERISCOCARDIOVASCULARES}

\section{a) Estudos clínicos envolvendo seres humanos}

Vários estudos têm verificado a relação entre índice glicêmico e prevenção ou melhora dos parâmetros que predispõem o desenvolvimento de doenças cardiovasculares. Muitos desses estudos, principalmente aqueles conduzidos com humanos de vida livre, estão sujeitos à interferência de vários fatores, de difícil controle e que podem interferir e confundir os resultados obtidos.

O estudo de Lukaczer et al. ${ }^{33}$ avaliou o efeito de um programa dietético combinando alimentos de baixo índice glicêmico com proteína de soja e isoflavona na diminuição dos fatores de risco para desenvolvimento de doenças cardiovasculares, em mulheres na pós-menopausa. Durante um período de 12 semanas, 27 mulheres foram orientadas a ingerir alimentos com baixo índice glicêmico associada à ingestão de $30 \mathrm{~g}$ de proteína de soja (isoflavona) e $4 \mathrm{~g}$ de fitosteróis. Outras 26 foram orientadas a seguir um programa de dieta padrão da American Heart Association Step 1. Ambos os grupos possuíam idade, Índice de Massa Corporal (IMC), pressão arterial e LDL-colesterol similares. Os resultados mostraram que o grupo que ingeriu a dieta contendo alimentos de baixo índice glicêmico apresentou maior redução dos seguintes fatores de risco cardiovascular: diminuição das concentrações de colesterol total, LDL-colesterol, triglicerídeos, hemoglobina glicada, homocisteína e dos valores de pressão arterial, enquanto que as concentrações de HDL-colesterol aumentaram.

Entretanto, não é possível afirmar se tais resultados foram devidos à ingestão de alimentos de baixo índice glicêmico ou de fitosteróis e de isoflavonas, já que estes dois últimos componentes têm demonstrado efeito sobre a melhora no perfil lipídico de indivíduos com dislipidemias ${ }^{34,35}$. Além disso, o grupo que ingeriu alimentos de baixo índice glicêmico também apresentou menor consumo médio de energia e maior perda de peso, durante o estudo (em torno de $8 \mathrm{~kg}$, contra $3,8 \mathrm{~kg}$ do outro grupo $^{33}$. Sabe-se que o excesso de peso pode ser relacionado à resistência insulínica e que a redução do peso corporal pode aumentar a sensibilidade insulínica, melhorando assim o perfil lipídico ${ }^{5,36-38}$. Apesar de os autores daquele estudo ${ }^{33}$ não terem 
indicado o tipo de fibra ingerida (solúvel ou insolúvel), deve-se destacar que houve um maior consumo no grupo que ingeriu alimentos de baixo índice glicêmico. Os resultados de alguns estudos sugerem a possível ação das fibras na redução do colesterol sangüíneo ${ }^{3,39,40}$. Assim, pode-se perceber que a ausência do controle sobre todos esses fatores coloca em dúvida o possível efeito do índice glicêmico nos parâmetros avaliados.

Em outro estudo randomizado, apresentando delineamento em cross-over, Jiménes-Cruz et al. ${ }^{22}$ avaliaram o efeito do índice glicêmico de duas dietas mexicanas mistas, na glicemia de jejum, no LDL-colesterol, HDL-colesterol, colesterol total e nos triglicerídeos em indivíduos com diabetes mellitus do tipo 2. Durante três semanas, 8 indivíduos com diabetes há 7 (desvio-padrão $\mathrm{DP}=6)$ anos, com idade de 51 ( $\mathrm{DP}=3$ ) anos, ingeriram as dietas de alto (72) e moderado (60) índice glicêmico, em condições de vida livre. Os resultados do estudo demonstraram que durante o período de consumo de alimentos de moderado índice glicêmico, houve redução significante do LDL-colesterol e do colesterol total desses indivíduos, sem que houvesse alterações na ingestão energética e no peso corporal.

No entanto, naquele estudo ${ }^{22}$, o maior consumo de fibras durante o período de ingestão da dieta de moderado índice glicêmico, mais uma vez, comprometeu a atribuição do efeito do índice glicêmico nos parâmetros avaliados. Enquanto esta dieta continha 30 g/dia ( $\mathrm{DP}=0,7 \mathrm{~g}$ de fibra alimentar), a dieta de baixo índice glicêmico resultava em uma ingestão de fibras equivalente a $53 \mathrm{~g} / \mathrm{dia}$ $(D P=0,6)$, valor este muito superior às recomendações. Os resultados de alguns estudos indicam que a maior ingestão de fibras pode ser responsável pela diminuição do colesterol total e do LDL-colesterol ${ }^{3,39-41}$. Além disso, a ingestão de carboidratos durante o período de consumo de alimentos de moderado índice glicêmico foi significantemente menor e, conseqüentemente, a carga glicêmica também ${ }^{22}$. Este resultado sugere que, conforme as recomendações da ADA, o controle da quantidade do carboidrato consumido pelo indivíduo diabético pode ser mais importante na melhora dos parâmetros bioquímicos, do que a qualidade do carboidrato ingerido.

Por outro lado, estudos bem controlados observaram benefícios em função da utilização do índice glicêmico. $O$ estudo de Frost et al..$^{13}$ avaliou a influência do índice glicêmico na sensibilidade insulínica do adipócito ou do organismo como um todo, bem como a influência deste índice na produção de TNF- $\alpha$ em mulheres com risco de doença coronariana. Um total de 28 mulheres, sendo 16 com história familiar de doença coronariana, consumiram, de forma randomizada, dieta isoenergética de baixo ou alto índice glicêmico, apresentando proporção de macronutrientes e teor de fibras semelhantes, por três semanas. Foi incluído no estudo outro grupo de 33 mulheres, sendo 8 com história familiar de doença coronariana, as quais ingeriram dieta habitual durantes as três semanas. Foi possível verificar que tanto ao início quanto ao término do estudo não havia diferença na idade, no IMC, na relação cintura-quadril, na glicemia e lipemia de jejum das participantes. Entretanto, a insulinemia de jejum foi maior nas mulheres com risco de doença coronariana que consumiram a dieta habitual. Ao final do estudo, verificou-se que houve aumento da sensibilidade insulínica do adipócito nas mulheres com risco de doença coronariana que consumiram dieta de baixo índice glicêmico. Os resultados desse estudo demonstraram o possível benefício a curto prazo do consumo de alimentos de baixo índice glicêmico na redução do risco de desenvolvimento de doenças cardiovasculares, devido à melhora da sensibilidade insulínica.

Em outro estudo ${ }^{16}, 23$ indivíduos obesos foram aleatoriamente designados para ingerir à vontade, durante 12 meses, 2 tipos distintos de dieta - de baixo índice glicêmico e baixa carga glicêmica (45\%-55\% de carboidratos) ou dieta convencional para perda de peso (restrição de 250-500 kcal/dia) e redução do risco de doenças cardiovasculares (55\%-60\% carboidratos). Apesar de a dieta de baixa carga glicêmica não ter sido 
planejada inicialmente para que houvesse diminuição da ingestão energética, esta ocorreu na mesma proporção que a convencional. Ao final do estudo, foi possível verificar que, apesar de ambos os grupos apresentarem redução nas concentrações de triglicerídeos e do inibidor do ativador de plasminogênio 1 (PAI-1), os indivíduos que ingeriram dieta com baixa carga glicêmica apresentaram maiores reduções em tais parâmetros. Esse resultado indica que tanto a quantidade, quanto a qualidade do carboidrato ingerido (refletidos na carga glicêmica) exerceram um efeito positivo na redução desses 2 últimos parâmetros citados.

\section{b) Estudos clínicos envolvendo animais}

A condução de estudo envolvendo animais de laboratório é de mais fácil execução, uma vez que o pesquisador tem possibilidade de controlar todos os fatores que podem interferir nos resultados. Tais estudos podem ser conduzidos durante todo o ciclo de vida do animal e são menos onerosos. No entanto, deve-se considerar que em função de diferenças na fisiologia e no metabolismo, os resultados obtidos em tais estudos nem sempre podem ser extrapolados para seres humanos.

Em um desses estudos ${ }^{42}$, foram avaliados em 40 ratos os efeitos da ingestão de duas dietas isoenergéticas, isoglicídicas e isolipídicas, contendo $57,5 \%$ de carboidrato proveniente de feijão (baixo índice glicêmico) ou de amido de milho (alto índice glicêmico), nas concentrações de leptina plasmática, glicemia, insulinemia e perfil lipídico, após 3 e 12 semanas. Os animais foram treinados a ingerirem a dieta oferecida em 15 minutos, semelhante ao procedimento adotado com humanos, em estudos para determinação do índice glicêmico.

Os resultados demonstraram que as áreas abaixo da curva e os picos de resposta glicêmica e insulinêmica, 60 minutos após a ingestão das dietas, foram maiores nos ratos que consumiam dieta com alto índice glicêmico. A ingestão alimentar, o peso corporal, as concentrações basais de insulina, glicose e triglicerídeos não diferiram entre os dois grupos. Os níveis de mRNA do gene ob diminuiu em $50 \%$, após 3 semanas de ingestão da dieta com alto índice glicêmico, sem que houvesse redução significante da leptina plasmática. Após 12 semanas de ingestão desta dieta, houve redução significante tanto da leptina plasmática, quanto do mRNA do gene ob. No entanto, não foi verificada alteração no teor de gordura corporal com a ingestão da dieta de alto índice glicêmico. As concentrações de ácidos graxos livres se elevaram significantemente e se correlacionaram negativamente com a leptina plasmática. Apesar das baixas concentrações de leptina, os ratos que ingeriram dietas de alto índice glicêmico não aumentaram a ingestão alimentar, sugerindo a ocorrência de redução na sensibilidade à leptina. Segundo os autores do estudo ${ }^{42}$, esses resultados podem preceder o ganho de peso e o aumento da massa gordurosa.

Em outro estudo ${ }^{43}$, avaliou-se o efeito do índice glicêmico na adiposidade, homeostasia da glicose e lipídeos plasmáticos, após oferecimento de dietas apresentando conteúdo idêntico de fibras, macro e micronutrientes, palatabilidade semelhante, diferindo apenas na composição do amido. Ratos parcialmente pancreatectomizados foram aleatoriamente alocados em dois grupos, em que dietas de alto (542 g/kg de amido, 100\% composto por amilopectina) ou baixo $(542 \mathrm{~g} / \mathrm{kg}$ de amido, $60 \%$ de amilose e $40 \%$ de amilopectina) índice glicêmico foram ingeridas, por um período de 18 semanas. A composição de tais dietas se baseou no fato que a amilopectina apresenta maior efeito na elevação do índice glicêmico do que a amilose. Os animais que consumiram dieta de alto índice glicêmico apresentaram maior área abaixo da curva da resposta glicêmica e insulinêmica. Na sétima semana do estudo, esses animais também apresentaram concentração plasmática de triglicerídeos três vezes maior. Ao final do estudo, verificou-se que apesar de o ganho de peso ter sido semelhante em ambos os grupos, os animais que consumiram dieta de alto índice glicêmico apresentaram $71 \%$ a mais de gordura corporal e $8 \%$ menos de massa magra que os do 
outro grupo. Após autópsia, verificou-se ainda que os animais com dieta de alto índice glicêmico apresentavam maior teor de gordura epididimal e retroperitoneal. Sabe-se que o aumento da adiposidade favorece o desenvolvimento de resistência insulínica e aumenta a expressão de marcadores inflamatórios, estando estes dois fatores diretamente ligados ao desenvolvimento de doenças cardiovasculares ${ }^{37,38}$.

\section{c) Estudos epidemiológicos}

O impacto do índice glicêmico e da carga glicêmica nas doenças cardiovasculares também tem sido avaliado em estudos epidemiológicos. Nesses estudos, índice glicêmico e a carga glicêmica da dieta ingerida por uma determinada população são avaliados a partir de dados obtidos por meio de recordatórios dietéticos e questionários de freqüência alimentar, os quais são métodos simples e rápidos para se obter as informações necessárias, de modo transversal15,19,31.

Em um desses estudos ${ }^{31}$, utilizou-se o recordatório de três dias para avaliar o índice glicêmico da dieta consumida por 32 mulheres japonesas, e a partir daí avaliar a correlação entre índice glicêmico e fatores de risco (peso, IMC, gordura corporal, triglicerídeos, HDL-colesterol, LDL-colesterol, glicemia de jejum e insulina) para manifestação de doenças cardiovasculares. Após ajuste para ingestão energética, ingestão diária de fibras, idade e tempo gasto em atividade física, verificou-se a ocorrência de maiores concentrações de HDLcolesterol e menores concentrações de insulina no menor tercil de índice glicêmico e carga glicêmica.

É interessante ressaltar que os indivíduos do menor tercil de índice glicêmico e carga glicêmica também consumiam mais gordura total, principalmente as monoinsaturadas, e menor quantidade de carboidratos. Tais características dietéticas têm sido associadas ao menor risco de desenvolvimento de doenças cardiovasculares $^{5,13,44,45}$. Dessa forma, os resultados obtidos nesse estudo ${ }^{31}$ não podem ser atribuídos apenas ao baixo índice glicêmico e à carga glicêmica da dieta ingerida. Mais uma vez, os resultados desse estudo sugerem que tanto a quantidade, quanto a qualidade do carboidrato ingerido podem ser importantes para tratamento e prevenção de doenças cardiovasculares.

Além disso, deve-se destacar que naquele estudo ${ }^{31}$, o cálculo do índice glicêmico e da carga glicêmica foi feito considerando apenas os alimentos que continham mais de um grama de carboidrato na porção. Assim, alimentos como carnes, ovos e leites, os quais possuem baixos valores de índice glicêmico, não foram considerados. No entanto, deve-se ressaltar que tais alimentos protéicos afetam o índice glicêmico de refeições mistas, por interferir na taxa de esvaziamento gástrico, absorção e liberação de insulina 25,30,46.

Além das proteínas, as gorduras, alguns antinutrientes e a acidez podem alterar o esvaziamento gástrico e a secreção insulínica, afetando o índice glicêmico de dada refeição composta por uma série de alimentos ${ }^{27,47}$. As gorduras e as fibras presentes nos alimentos diminuem a velocidade de esvaziamento gástrico ${ }^{43}$. As fibras, ainda, aumentam o trânsito intestinal. Dessa forma, a determinação do índice glicêmico de uma refeição mista não deve ser feita considerando o índice glicêmico dos alimentos isolados ${ }^{13}$. Diante disso, conclui-se que uma das grandes limitações da utilização de inquéritos para cálculo do índice glicêmico e da carga glicêmica se refere ao fato de que tais instrumentos não consideram as interações ocorridas entre os alimentos que compõem as refeições mistas, já que se ingere um conjunto de alimentos na maioria das refeições feitas diariamente.

Buyken et al. ${ }^{48}$ também utilizaram recordatório de três dias para avaliar a relação entre índice glicêmico e hemoglobina glicada e lipídeos séricos. Esse estudo, de natureza transversal, envolveu 2746 diabéticos do tipo 1, com idade entre 15-60 anos. Os resultados demonstraram que após ajuste para eliminar o efeito de fatores interferentes (ingestão energética, consumo de fibras e álcool, gênero, idade, presença de sobre- 
peso e hábito de fumar), houve correlação positiva entre a hemoglobina glicada e o índice glicêmico. Houve ainda redução do HDL-colesterol e aumento de triglicerídeos com o aumento do índice glicêmico. Entretanto, nesse estudo foram utilizados dados obtidos apartir de recordatórios, os quais podem não refletir fielmente a dieta normalmente consumida.

Além do mais, os resultados daquele estudo ${ }^{48}$ foram apresentados em quartis. Para o primeiro quartil, considerou-se o valor médio de índice glicêmico correspondente a 74,9 e para o quarto quartil, 88,5. Alguns autores ${ }^{49}$ sugerem a utilização dos seguintes pontos de corte para classificar os alimentos quanto ao índice glicêmico: alto índice glicêmico $\geq 69$; moderado índice glicêmico =56-69 e baixo índice glicêmico $\leq 56$. Dessa forma, todos os quartis apresentaram índice glicêmico elevado.

\section{CONSIDERAÇÕ ES FIN A IS}

A utilização do índice glicêmico na prevenção e no controle de doenças cardiovasculares ou na melhora de seus fatores de risco permanece controversa. Muitas dessas controvérsias refletem problemas metodológicos apresentados pelos estudos até então conduzidos. A condução de mais estudos clínicos de longa duração, apresentando delineamento experimental adequado, envolvendo humanos, ainda é necessária para chegar a uma conclusão a respeito do efeito da qualidade (índice glicêmico) do carboidrato ingerido na manifestação e no tratamento dessas doenças. Dessa forma, conforme preconizado pela American Diabetes Association, o controle da quantidade do carboidrato ingerido parece, no momento, ser mais eficaz.

\section{COLABORADORES}

G.Q. CARVALHO e R.C.G. ALFENAS responsáveis pela revisão bibliográfica do conteúdo, pela redação do artigo, pela análise crítica dos estudos utilizados e pela correção final do artigo.

\section{REFERÊ NCIAS}

1. Cervato AM, Mazzilli RN, Martins IS, Marucci MFN Dieta habitual e fatores de risco para doenças cardiovasculares. Rev Saúde Pública. 1997; 31(3): 227-35.

2. Lotufo PA. Mortalidade precoce por doenças do coração no Brasil. Comparação com outros países. Arq Bras Cardiol. 1998; 70(5):321-5.

3. Rique $A B R$, Soares EA, Meirelles CM. Nutrição e exercício na prevenção e controle das doenças cardiovasculares. Rev Bras Med Esporte. 2002; 8(6): 244-54.

4. Santos Filho RD, Martinez TLR. Fatores de risco para doença cardiovascular: velhos e novos fatores de risco, velhos problemas! Arq Bras Endocrinol Metabol. 2002; 46(3):212-4.

5. Murphy NF, Simpson CR, MacIntyre K, McAlister FA, Chalmers J, McMurray JJV. Prevalence, incidence, primary care burden and medical treatment of angina in Scotland: age, sex and socioeconomic disparities: a population-based study. Heart. 2006; 92(8):1047-54.

6. Fox K, Garcia MA, Ardissino D, Buszman P, Camici $P G, C r e a$ F, et al. Task force on the management of stable angina pectoris of the european society of cardiology; ESC Committee for Practice Guidelines (CPG). Guidelines on the management of stable angina pectoris: executive summary. The task force on the management of stable angina pectoris of the european society of cardiology. Eur Heart J. 2006; 27(11):1341-81.

7. Santos CRB, Portella ES, Ávila SS, Soares EA. Fatores dietéticos na prevenção e tratamento de comorbidades associadas à síndrome metabólica. Rev Nutr. 2006; 19(3):389-401.

8. International Diabetes Federation. The IDF consensus worldwide definition of the metabolic syndrome, 2006 [cited 2007 Dec 17]. Available from: <http://www.idf.org/webdata/docs/IDF_ Meta_def_final.pdf>.

9. Popkin BM. The nutrition transition and obesity in the developing world. J Nutr. 2001; 131(3): 871s-3s

10. Pearson TA, Blair SN, Daniels SR, Eckel RH, Fair JM Fortmann SP, et al. AHA Guidelines for primary prevention of cardiovascular disease and stroke: 2002 update consensus panel guide to comprehensive risk reduction for adult patients without coronary or other atherosclerotic vascular diseases. Circulation. 2002; 106(3):388-91.

11. Departamento de Aterosclerose da Sociedade Brasileira de Cardiologia. IV Diretriz brasileira sobre dislipidemias e prevenção da aterosclerose. Arq Bras Cardiol. 2007; 88(Supl.1):2s-19s. 
12. Roos NM, Bots ML, Katan MB. Replacement of dietary satured fatty acids by trans fatty acids lowers serum HDL cholesterol and impairs endothelial function in healthy men and women. Arterioscler Thromb Vasc Biol. 2001; 1(7):1233.

13. Frost G, Leeds A, Trew G, Margara R, Dornhorst A. Insulin sensitivity in women at risk of coronary heart disease and the effect of a low glycemic diet. Metabolism. 1998; 47(10):1245-51.

14. Oh K, Hu FB, Cho E, Rexrode KM, Stampfer MJ, Manson JE, et al. Carbohydrate intake, glycemic index, glycemic load, and dietary fiber in relation to risk of stroke in women. Am J Epidemiol. 2005; 161(2):161-9.

15. Liu S, Manson JE, Stampfer MF, Holmes MD, Hu FB, Hankinson SE, et al. Dietary glycemic load assessed by food-frequency questionnaire in relation to plasma high-density-lipoprotein cholesterol and fasting plasma triacylglycerols in postmenopausal women. Am J Clin Nutr. 2001; 73(3):560-6.

16. Ebbeling $C B$, Leidig MM, Sinclair KB, SegerShippee LG, Feldman HA, Ludwig DS. Effects of an ad libitum low-glycemic load diet on cardiovascular disease risk factors in obese young adults. Am J Clin Nutr. 2005; 81(5):976-82.

17. Beebe C. Diets with a low glycemic index: not ready for practice yet! Nutr Today. 1999; 34(2):82-6.

18. Franz MJ. In defense of the American Diabetes Association's recomendations on the glycemic index. Nutr Today. 1999; 34(2):78-81.

19. Olendzki BC, Ma Y, Culver AL, Pharm BS, Ockene IS, Griffith JA, et al. Methodology for adding glycemic index and glycemic load values to $24-$ hour dietary recall database. Nutrition. 2006; 22(11-12):1087-95.

20. Jenkins DJA, Kendall CWW, Augustin LSA, Franceschi S, Hamidi M, Marchie A, et al. Glycemic index: overview of implications in health and disease. Am J Clin Nutr. 2002; 76(Suppl):266S-73S.

21. Leeds AR. Glycemic index and heart disease. Am J Clin Nutr. 2002; 7(1):286s-9s.

22. Jiménez-Cruz A, Turnbull WH, Bacardi-Gascón M, Rosales-Garay P. A high-fiber, moderate-glycemicindex, Mexican style diet improves dyslipidemia in individuals with type 2 diabetes. Nutr Res. 2004; 24(1):19-27.

23. Sahyoun NR, Anderson AL, Kanaya AM, KohBanerjee P, Kritchevsky SB, Rekeneire N, et al. Dietary glycemic index and load, measures of glucose metabolism, and body fat distribution in older adults. Am J Clin Nutr. 2005; 82(3):547-52.

24. Wolever TMS, Mehling C. Long-term effect of varying the source or amount of dietary carbohydrate on postprandial plasma glucose, insulin, triacylglycerol, and free fatty acid concentrations in subjects with impaired glucose tolerance. Am J Clin Nutr. 2003; 77(3):612-21.

25. Pi-Sunyer FX. Glycemic index and disease. Am J Clin Nutr. 2002; 76(Suppl):290S-8S.

26. França HH. O paradoxo da doença coronariana. Arq Bras Cardiol. 2002; 79(4):419-21.

27. Laville M. Could glycaemic index be the basis of simple nutritional recommendations? Br J Nutr. 2004; 91(6):803-4.

28. Brand-Miller J, Foster-Powell K. Diets with a low glycemic index: from theory to practice. Nutr Today. 1999; 34(2):64-72.

29. Sartorelli DS, Cardoso MA. Associação entre carboidratos da dieta habitual e diabetes mellitus tipo 2: evidências epidemiológicas. Arq Bras Endocrinol Metabol. 2006; 50(3):415-25.

30. Sheard NF, Clark NG, Brand-Miller JC, Franz MJ, PiSunyer FX, Mayer-Davis E, et al. Dietary carboidrate (amount and type) in the prevention and management of diabetes. A statement by the American Diabetes Association. Diabetes Care. 2004; 27(9):2266-70.

31. Amano Y, Kawakubo K, Lee JS, Tang AC, Sugiyama M, Mari K. Correlation between dietary glycemic index and cardiovascular disease risk factors among Japanese women. Eur J Clin Nutr. 2004; 58(11): 1472-8.

32. Brand-Miller JC. Postprandial glycemia, glycemic index, and the prevention of type 2 diabetes. Am J Clin Nutr. 2004; 80(2):243-4.

33. Lukaczer D, Liska DJ, Lerman RH, Darland G, Schiltz $B$, Tripp $M$, et al. Effect of a low glycemic index diet with soy protein and phytosterols CVD risk factors in postmenopausal women. Nutrition. 2006; 22(2):104-13.

34. Erdman Jr. JW. For the AHA Nutrition Comittee. Soy protein and cardiovascular disease: a statement for healthcare professionals from the Nutrition Comittee of the AHA. Circulation. 2000; 102(20): 2555-9.

35. Moghadasian MH, Frolich JJ. Effects of dietary phytosterols on cholesterol metabolism and atherosclerosis: clinical and experimental evidence. Am J Med. 1999; 107(6):588-94.

36. Hamdy O, Ledbury S, Mullooly C, Jarema C, Porter $S$, Ovalle $K$, et al. Lifestyle modification improves endothelial function in obese subjects with the insulin resistance syndrome. Diabetes Care. 2003; 26(7):2119-25.

37. Hermsdorff HHM, Monteiro JBR. Gordura visceral, subcutânea ou intramuscular: onde está o problema? Arq Bras Endocrinol Metabol. 2004; 48(6): 803-11. 
38. Ruderman N, Chisholm D, Pi-Sunyer X, Schneider $S$. The metabolically obese, normal-weight individual revisited. Diabetes. 1998; 47(5):699-713.

39. Davidson MH, Dugan LD, Burns JH, Bova J, Story K, Drennan KB. The hypocholesterolemic effects of beta-glucan in oatmeal and oatbran. A dosecontrolled study. JAMA. 1991; 265(14):1833-9.

40. Ripsin CM, Keenan JM, Jacobs DR, Elmer PJ, Welch RR, van Horn L, et al. Oat products and lipid lowering. A Meta-analysis. JAMA. 1992; 267(24): 3317-25.

41. Mekki N, Dubois C, Charbonnier M, Cara L, Senft $M$, Pauli AM, et al. Effects of lowering fat and increasing dietary fiber on fasting and postprandial plasma lipids in hypercholesterolemic subjects consuming a mixed Mediterranean-Western diet. Am J Clin Nutr. 1997; 66(6):1443-51.

42. Kabir M, Guerre-Millo M, Laromiguere M, Siama G, Rizkalla SW. Negative regulation of leptin by chronic high-glycemic index starch diet. Metabolism. 2000; 49(6):764-9.

43. Pawlak DB, Kushner JA, Ludwig DS. Effects of dietary glycaemic index on adiposity, glucose homoeostasis, and plasma lipids in animals. Lancet. 2004; 364(9436):778-85.

44. Bassand J. Managing cardiovascular risk in patients with metabolic syndrome. Clin Cornerstone. 2006; 8(Suppl 1):S7-S14.
45. Pitsavos C, Panagiostakos DB, Chrysosshoou C, Papaionnou I, Papadimitriou L, Tousoulis D, et al. The adoption of mediterranean diet attenuates the development of acute coronary syndromes in people with the metabolic syndrome. Nutr J. 2003; 2(1):7.

46. Flint A, Moller BK, Raben A, Pedersen D, Tetens I, Holst JJ, et al. The use of glycaemic index tables to predict glycaemic index of composite breakfast meals. Br J Nutr. 2004, 91(6):979-89.

47. Brouns F, Bjorck I, Frayn KN, Gibbs AL, Lang V, Slama $\mathrm{G}$, et al. Glycaemic index methodology. Nutr Res Rev. 2005; 18(1):145-71.

48. Buyken AE, Toeller M, Heitkamp G, Karamanos B, Rottiers R, Muggeo M, et al. Complications Study Group. Glycemic index in the diet of European outpatients with type 1 diabetes: relations to glycated hemoglobin and serum lipids. Am J Clin Nutr. 2001; 73(3):574-81.

49. Brand-Miller J, Foster-Powell K, Wolever TMS, Colagiuri S. The new glucose revolution: the authoritative guide to the glycemic index. New York: Merlowe \& Company; 2002.

Recebido em: 27/4/2007

Versão final reapresentada em: 8/1/2008

Aprovado em: 12/6/2008 